\title{
Neoformación digital
}

\section{Digital neoformation}

\author{
Miguel Ángel Cardona-Hernández, ${ }^{*}$ Maribet González-González, ${ }^{\ddagger}$ \\ Wendy Carolina González-Hernández ${ }^{\S}$
}

\section{CASO CLÍNICO}

El caso corresponde a un hombre de 62 años, originario y residente del Estado de México, sin antecedentes de importancia para su padecimiento actual.
Acude a consulta por una «lesión en el dedo», a decir de él, que ha crecido lentamente durante dos años. A la exploración física se observa una dermatosis localizada a la extremidad superior izquierda, la cual afectaba mano, y de ésta la cara dorsal del dedo me-

Figura 1:

A mayor acercamiento.

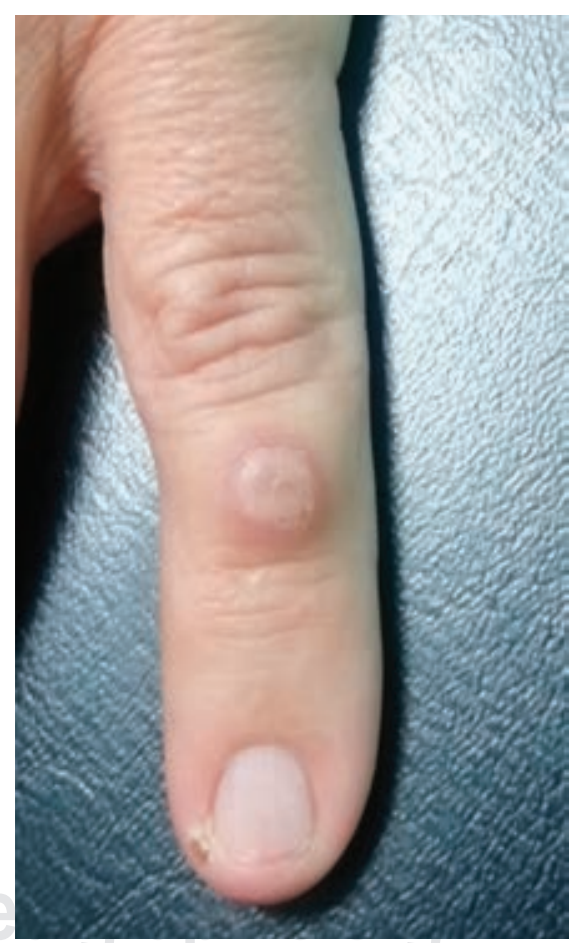

* Dermatólogo.

‡ Dermatopatóloga.

$\S$ Residente del tercer año de Dermatología.
Citar como: Cardona-Hernández MA, González-González M, González-Hernández WC. Neoformación digital. Rev Cent Dermatol Pascua. 2020; 29 (2-3): 90-93. https:// dx.doi.org/10.35366/99922

Centro Dermatológico «Dr. Ladislao de la Pascua», SSCDMX. 


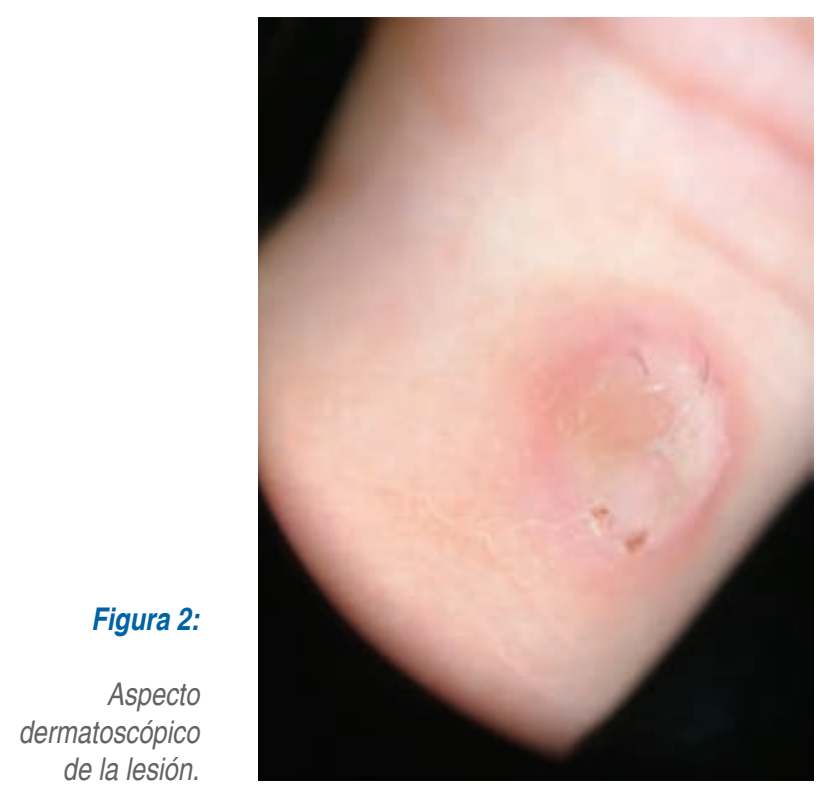

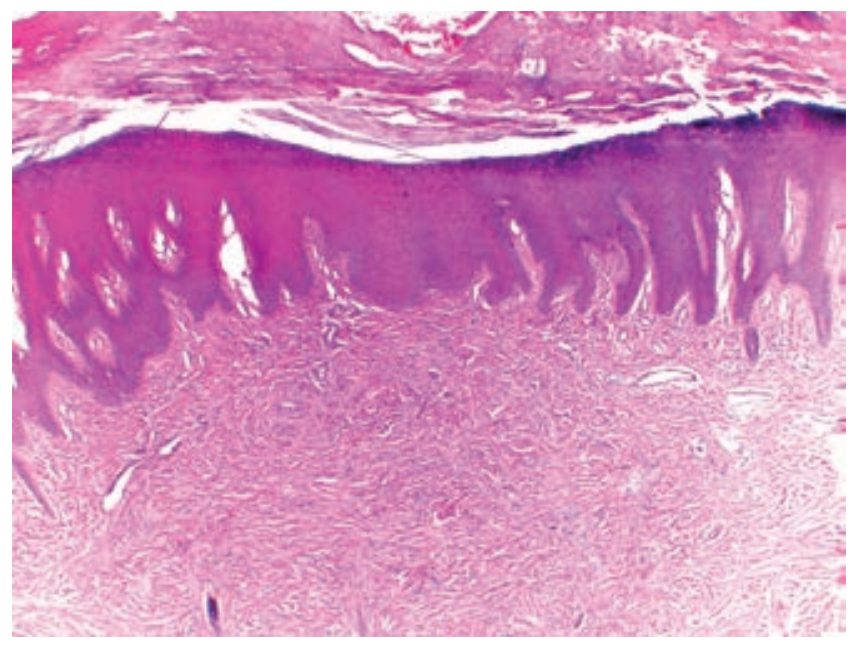

Figura 3: Epidermis con hiperqueratosis, hipergranulosis y acantosis regular. En el espesor de la dermis se observa una neoformación. $H \& E 4 x$.

ñique, a nivel de la falange media, constituida por una neoformación exofítica, oval, de aspecto queratósico, de $1 \times 0.8 \mathrm{~cm}$, de bordes bien definidos y superficie anfractuosa en algunas áreas. De evolución crónica y asintomática (Figura 1). A la dermatoscopía con luz polarizada se observa una neoformación con áreas blanquecinas centrales y escama gruesa, con eritema en su periferia (Figura 2). No había recibido tratamiento previo. Con la sospecha de un probable tumor de anexos se realiza biopsia excisional, la cual

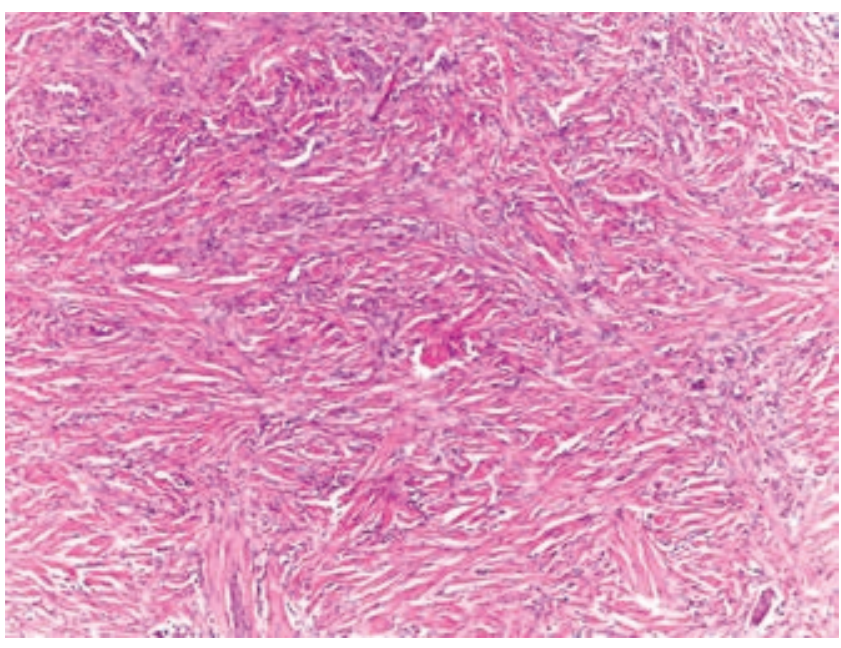

Figura 4: Neoformación constituida por células fusiformes entremezcladas con fibras de colágeno gruesa que siguen diversos trayectos. H\&E 10x.

reporta: epidermis con hiperqueratosis, paraqueratosis focal, hipergranulosis y zonas con acantosis regular moderada (Figura 3), en el espesor de la dermis se observan células de núcleo fusiforme, y otras de tipo epitelioide, las cuales se entremezclan con fibras de colágena gruesa; se disponen en haces y siguen diversos trayectos (Figura 4).

Con los datos proporcionados, ¿cuál es su diagnóstico? 


\section{DIAGNÓSTICO: DERMATOFIBROMA}

El dermatofibroma, también conocido como «histiocitoma cutáneo fibroso", pertenece al grupo de neoformaciones benignas de tejidos blandos más frecuentes. ${ }^{1}$

Antes del año 2003 no se habían reportado casos en topografía digital, considerándose en la actualidad una localización muy poco frecuente. ${ }^{2}$

Por lo general, se desarrolla en mujeres entre la segunda y tercera décadas de la vida, comprometiendo tronco y extremidades, tanto superiores como inferiores. Sin embargo, existen comunicaciones de casos desarrollados a cualquier edad y en topografías poco frecuentes, como falanges, palmas, plantas, piel cabelluda y cara. Específicamente, la afectación digital se ha observado hasta en $2 \%$ de algunas series de casos, en pacientes de nueve hasta 70 años, predominando en hombres en una proporción 2:1.3-7

Kyung-Hwa y colaboradores detectaron una concordancia entre la localización de estas lesiones a nivel digital y la mano dominante en los individuos afectados $(83.3 \%) .{ }^{4}$ Ellos observaron que se manifestaba como una neoformación de aspecto nodular, circunscrita y firme, hiperpigmentada o no, de aproximadamente $0.5 \mathrm{a}$ $1 \mathrm{~cm}$ de diámetro, de evolución crónica (en promedio de 11.2 meses), asintomática o con ligero dolor al roce..$^{2,4}$ En cuatro pacientes el dedo involucrado fue el medio, en tres el dedo índice y en uno el pulgar. La ubicación dorsal fue la más común (50\%). ${ }^{4}$

Lehmer y colaboradores reportaron una serie de 26 casos de dermatofibromas de localización digital, afectando a personas de 27 a 70 años, con una proporción de afectación hombre-mujer de 2-2.5: ${ }^{5}$

Su fisiopatogenia es aún desconocida, sin embargo, cuando las lesiones se ubican en las falanges, se le ha relacionado con procesos reactivos-reparadores desencadenados por traumatismos ${ }^{3,6}$ (sobre todo por picaduras de insectos), lo cual induce una proliferación benigna de fibroblastos. ${ }^{1,2}$

De manera habitual, se manifiesta como una neoformación de consistencia firme, hemiesférica, generalmente hiperpigmentada, de alrededor de 0.5 a $1 \mathrm{~cm}$ de diámetro, localizada en extremidades inferiores 0 tronco. En general es asintomática, ${ }^{1}$ sin embargo, en algunas ocasiones los pacientes refieren prurito o dolor a la palpación. ${ }^{2}$ En algunos casos estos tumores crecen rápidamente ${ }^{8}$ aunque la mayoría de ellos cursa con una evolución estacionaria, sin cambios durante años. ${ }^{4} \mathrm{~A}$ la palpación se puede observar el «signo de la pastilla», en el cual la neoformación se deprime al comprimir sus caras laterales entre dos dedos, pero la presencia de este signo no es de utilidad cuando se presentan lesiones a nivel digital. .,5,9

El diagnóstico se basa principalmente en las características clínicas y el interrogatorio. A la exploración dermatoscópica se observa una red de pigmento periférica, con un área cicatrizal central. ${ }^{6}$ La biopsia cutánea se realiza en los casos en los que el diagnóstico clínico es dudoso. ${ }^{7}$

Entre los diagnósticos diferenciales se incluyen: nevo melanocítico, carcinoma basocelular, queratoacantoma y dermatofibrosarcoma protuberans. ${ }^{8}$

\section{Histopatología}

El estudio histopatológico muestra una epidermis hiperplásica e hiperpigmentada. A nivel de la dermis, se aprecian células fusiformes sin atipias, dispuestas en fascículos elongados paralelos a la epidermis, que están rodeados por estroma colagenoso o, menos frecuentemente, mixoide. En algunas ocasiones también se pueden observar histiocitos espumosos, células gigantes multinucleadas, infiltrados focales de células inflamatorias y hemosiderina. En la periferia de la lesión característicamente se observan haces individuales de colágena hialina rodeados por células tumorales., ${ }^{9,10}$ Estos hallazgos se extienden hasta el tejido celular subcutáneo.

Dentro del diagnóstico diferencial histopatológico se deben considerar los siguientes tumores: perineuroma, fibromixoma acral superficial, fibroqueratoma acral y dermatofibrosarcoma protuberans acral, ${ }^{6,10,11}$ siendo este último el más importante.

El estudio por inmunohistoquímica muestra positividad para el factor XIIla y vimentina, siendo CD34 negativo, a diferencia del dermatofibrosarcoma protuberans. ${ }^{9,10}$ También se han detectado otros marcadores útiles para su diferenciación como: vimentina, actina músculo específica, KP-1 y HAM56. ${ }^{6}$

En especial en los casos en los que el dermatofibroma se ubica a nivel digital, se debe hacer diagnóstico diferencial con un tumor de la infancia llamado «fibromatosis digital infantil», el cual aparece casi exclusivamente en los dedos, se desarrolla en el primer año de vida y se manifiesta como neoformaciones del color de la piel, ocasionando en algunos casos deformidad y disfunción motriz, llegando a presentar regresión espontánea en meses 0 años posteriores. ${ }^{11-14}$

La falta de familiarización con esta topografía puede resultar en tratamientos agresivos innecesarios. No se requiere de alguno en especial, excepto que la lesión sea sintomática, siendo la extirpación quirúrgica el trata- 
miento de elección. Se han descrito otras alternativas de tratamiento como la criocirugía, la cual ha demostrado resultados variables. ${ }^{15}$

La recurrencia clínica en estos tumores es poco frecuente si se extirpa completamente. ${ }^{12}$

\section{COMENTARIO}

Se comunica este caso debido a la topografía y morfología poco frecuentes, comparadas con los dermatofibromas comunes. Es necesario realizar un estudio histopatológico ante la duda diagnóstica. El dermatofibroma se debe añadir a la lista de diagnósticos diferenciales de neoformaciones circunscritas en topografía digital.

Lo poco frecuente de la topografía digital en esta patología podría resultar en tratamientos agresivos innecesarios, por lo que es preciso establecer un diagnóstico certero.

\section{REFERENCIAS}

1. Goldsmith LA, Katz SI, Gilchrest BA, Paller AS, Leffell DJ, Wolff K et al. Fitzpatrick dermatología en medicina general. 8a ed. Buenos Aires; Editorial Médica Panamericana. 2014. p. 710.

2. Yamamoto T, Umeda T, Nishioka K. Dermatofibroma of the digit: report of six cases. Dermatology. 2003; 207: 79-81.

3. Mentzel T. Cutaneous mesenchymal tumours: an update. Pathology. 2014; 46: 149-159.

4. Kyung-Hwa N, Sang-Woo P, Seok-Kweon Y. A clinicohistopathological analysis of cutaneous fibrous histiocytomas of the finger. Indian $\mathrm{J}$ Dermatol. 2020; 65: 401-405.

5. Lehmer L, Ragsdale B. Digital dermatofibromas-common lesion, uncommon location: a series of 26 cases and review of the literature. Dermatol Online J. 2011; 17: 2.
6. Hernández-Salazar A, Saeb-Lima M, Domínguez-Cherit J Dermatofibroma en dedos de las manos. Piel. 2012; 27: 416-422.

7. Ramesh R, Jenkins R. Digital dermatofibroma: common lesion, uncommon site. J Am Acad Dermatol. 2013; 68 (Suppl. 1): AB48.

8. Requena L, Fariña MC, Fuente C, Piqué E, Olivares M, Martín $L$ et al. Giant dermatofibroma. A little-known clinical variant of dermatofibroma. J Am Acad Dermatol. 1994; 30: 714-718.

9. Luzar B, Calonje E. Cutaneous fibrohistiocytic tumors an update. Histopathology. 2010; 56: 148-165.

10. Calonje E, Brenn T, Lazar A, McKee PH. Connective tissue tumors. En: Calonje JE, Brenn T, Lazar AJ, McKee PH. McKee's pathology of skin. 4th ed. China. Elsevier Saunders. 2012. p. 627

11. Weedon D. Fibrous tumors and tumor-like proliferations. En: Weedon D. Weedon's skin pathology. 3rd ed. Edinburg: Churchill Livingstone/ Elsevier. 2010, pp. 758-759.

12. Gaufin M, Michaelis T, Duffy K. Cellular dermatofibroma: clinicopathologic review of 218 cases of cellular dermatofibroma to determine the clinical recurrence rate. Dermatol Surg. 2019; 45: 1359-1364.

13. Laskin WB, Miettinen M, Fetsch JF. Infantile digital fibroma/ fibromatosis: a clinicopathologic and immunohistochemical study of 69 tumors from 57 patients with long-term follow-up. Am J Surg Pathol. 2009; 33: 1-13.

14. Taylor HO, Gellis SE, Schmidt BA, Upton J, Rogers GF. Infantile digital fibromatosis. Ann Plast Surg. 2008; 61: 472-476.

15. Lanigan S. Cryotherapy for dermatofibromas. Clin Exp Dermatol. 1987; 12: 121-123.

\author{
Correspondencia: \\ Dr. Miguel Ángel Cardona Hernández \\ Dr. Vértiz Núm. 464 Esq. Eje 3 Sur, \\ Col. Buenos Aires, 06780, \\ Alcaldía Cuauhtémoc, CDMX \\ Tel. 55 5634-9919 \\ E-mail: drmiguelcardona08@gmail.com
}

